\title{
Trendwende in der Organspende?
}

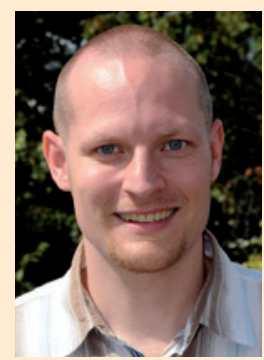

Christian Schäfer, Stuttgart
Für die über 10000 Menschen, die in Deutschland auf eine lebensrettende Organspende warten, ist es ein wichtiger Hoffnungsschimmer: Laut einer Statistik der Deutschen Stiftung Organtransplantation (DSO) hat sich die Zahl der Organspender im ersten Halbjahr 2015 erstmals seit Jahren stabilisiert bzw. im Vergleich zur selben Zeitspanne 2014 sogar um mehr als 6\% erhöht (ohne Lebendspender). Man muss zwar wegen des kurzen Zeitraums mit dem Begriff „Trendwende“ vorsichtig sein - daher das Fragezeichen in der Überschrift. Allerdings könnte diese Momentaufnahme tatsächlich der Auftakt zu einer beständigen Entwicklung sein, welcher die Organspende aus dem „Tal der Tränen“ führt. Vielleicht greifen nun endlich die Maßnahmen zur Qualitätskontrolle und Vertrauensbildung, die man umgesetzt hat, seit der Gesetzgeber das Transplantationsgesetz 2012 überarbeitet hat. Zur Erinnerung: Diese sind u.a.

- die Implementierung von Transplantationsbeauftragten in den Krankenhäusern,

- die Strafbarkeit von Manipulationen bei der Organallokation,

- unangekündigte, regelmäßige Kontrollen der Transplantationszentren bzw. Organentnahmekrankenhäuser,

- das Mehr-Augen-Prinzip bei der Entscheidung, ob ein Patient auf die Warteliste kommt (mindestens 3 Mediziner) und

- das 2-jährliche Verschicken von Organspendeausweisen durch die Krankenkassen an ihre Versicherten.

Wie die Ärzte Zeitung am 18. August berichtete, ist der Präsident der Deutschen Transplantationsgesellschaft (DTG) Prof. Björn Nashan, Hamburg, hier optimistisch: „Mit viel Aufklärung, Umsetzung der Gesetzesänderung und interdisziplinärer Zusammenarbeit gelingt vielleicht in 5-7 Jahren eine Verdoppelung der Spenderzahlen." Ich würde mich sehr freuen, wenn er Recht behielte - für die Menschen auf der Warteliste, von denen durchschnittlich 3 täglich sterben, wäre dies sicherlich noch mehr Grund zur Freude.

Natürlich besteht weiterhin Optimierungsbedarf: So erklärte der Medizinische Vorstand der DSO Dr. Axel Rahmel, Frankfurt am Main, gegenüber der Ärzte Zeitung, dass der Aufbau eines Transplantationsregisters die Sicherheit und Ergebnisqualität der Organspende nachhaltig verbessert könne und dies außerdem ein entscheidendes Element für die Transparenz sei. Auch meiner Meinung nach wäre diese Zentralisierung sehr wichtig - u.a. auch, um die Organzuordnung noch mehr an die Variabilität der Empfänger anzupassen und damit das Potenzial der vorhandenen Organe besser nutzen zu können.

Auch die Immunsuppression nach Organtransplantationen entwickelt sich ständig weiter. Um Ihnen einen aktuellen Überblick hierüber zu geben, ist diese Ausgabe der Dialyse aktuell dem Thema „Nierentransplantation und Immunsuppression" gewidmet: Ab Seite 361 können Sie die informativen Artikel zu der Thematik lesen und über den Fragebogen im Anschluss 3 CME-Punkte erwerben, so Sie denn Arzt sind. Pflegekräfte erhalten für das Abonnement bzw. den Bezug der Dialyse aktuell über eine Mitgliedschaft in der Arbeitsgemeinschaft für nephrologisches Personal e.V. (AfnP) oder dem Fachverband nephrologischer Berufsgruppen e.V. (fnb) jährlich 3 Punkte im Rahmen der „Registrierung beruflich Pflegender“. Ich wünsche Ihnen eine interessante Lektüre! 